\title{
APPLICATIONS OF THE CAUCHY INTEGRAL ON LIPSCHITZ CURVES
}

\author{
BY A. P. CALDERÓN ${ }^{1}$, C. P. CALDERÓN, E. FABES, M. JODEIT AND N. M. RIVIERE
}

Communicated by Hans Weinberger, April 25, 1977

We present here some new developments in the areas of partial differential equations and real analysis which have as their basis the following result. (See [2].)

THEOREM 1. Let $\Gamma$ be a curve in the complex plane given by the equation

$$
z(t)=t+i \varphi(t)
$$

where $\varphi(t)$ is a real valued function on the real line with a bounded derivative and let

$$
A_{\varphi, \epsilon} f=\frac{1}{2 \pi i} \int_{|s-t|>\epsilon} \frac{f(s)}{z(s)-z(t)} d z(s), \quad \epsilon>0
$$

then there exists a positive number a such that $\left\|\varphi^{\prime}\right\|_{\infty}<$ a implies that the operator $\sup _{\epsilon}\left|A_{\varphi, \epsilon} f\right|$ is of weak type $(1,1)$ and bounded in $L^{p}, 1<p<\infty$, and that $\lim _{\epsilon \rightarrow 0} A_{\varphi, \epsilon} f$ exists pointwise almost everywhere for $f \in L^{p}, 1 \leqslant p<\infty$.

Some of these applications were obtained independently by the authors, others were obtained jointly, and they will be published accordingly. However, because they are all closely related, the authors believed that they should be announced simultaneously. What follows is a sampling of these applications and the results presented are the most typical though not necessarily the most general ones.

\section{Singular integrals.}

THEOREM 2. Let $\Gamma$ be a simple closed curve in the complex plane with a continuously turning tangent. Let $f$ be a function on $\Gamma$ which is in $L^{p}$ with respect to arc length $1 \leqslant p<\infty$ and let

$$
F(z)=\frac{1}{2 \pi i} \int_{\Gamma} \frac{f(w)}{w-z} d w
$$

Then $F(z)$ has a limit when $z$ approaches $\Gamma$ nontangentially, almost everywhere with respect to arc-length, and the limit is a function in $L^{p}$ with respect to

AMS (MOS) subject classifications (1970). Primary 30-XX, 30A86, 44-XX. 44A25, $35 \mathrm{Hxx}, 35 \mathrm{~J} 25,31 \mathrm{Bxx}, 31 \mathrm{B2}$.

${ }^{1}$ A. P. Calderón is supported by NSF MCS75-05567.

Copyright $\odot$ 1978, American Mathematical Society 
arc-length provided that $1<p<\infty$. If the curve $\Gamma$ is merely rectifiable then the nontangential limit of $F(z)$ exists almost everywhere with respect to arc-length.

Concerning the behavior of the gradient of the Newtonian potential of mass distributions supported by surfaces we have the following result. (See also Theorem 7.)

THeORem 3. Let $\Phi(x)$ be a function in $\mathbf{R}^{n}$ which has a differential almost everywhere in the sense that $\Phi(x) \in T_{1}^{r}\left(x_{0}\right), 1 \leqslant r \leqslant \infty$ for almost all $x_{0} \in \mathbf{R}^{n}$. Then if $\Psi_{i} \in T_{1}^{p_{i}}\left(x_{0}\right), i=1,2, \ldots, m$, for almost all $x_{0} \in \mathbf{R}^{n}$ and if $f \in L^{q}$, $1 / r+\Sigma_{1}^{m} 1 / p_{i}+1 / q=1, m$ odd, the limit

$\lim _{\epsilon \rightarrow 0} \int_{|x-y|>\epsilon}\left[|x-y|^{2}+[\Phi(x)-\Phi(y)]^{2}\right]^{-(n+m) / 2} \prod_{1}^{m}\left[\Psi_{i}(x)-\Psi_{i}(y)\right] f(y) d y$ exists for almost all $x$.

The following may be considered as an extension of the theorem on commutators of R. R. Coifman and Y. Meyer.

THEOREM 4. Let $G\left(W_{1}, \ldots, W_{k}\right)$ be a function of the complex variables $W_{1}, \ldots, W_{k}$, holomorphic in $\left|W_{i}\right|<R_{i}$. Let $\Psi_{i}(x)$ be functions in $\mathbf{R}^{n}$ with distribution derivatives in $L^{p_{i}}, 1<p_{i} \leqslant \infty$, and $\varphi_{i}(x), i=1,2, \ldots, k$ functions with bounded derivatives. Let $k(x, z)$ be homogeneous of degree $-n$, with $\int_{|z|=1}|k(x, z)|^{s} d \sigma_{z}<\infty$, where $d \sigma_{z}$ is the surface area element of $|z|=1$. Then if $f \in L^{q}, 1<q \leqslant \infty$

$$
\begin{aligned}
\stackrel{*}{T}=\sup _{\epsilon} \mid \int_{|x-y|>\epsilon} \prod_{1}^{m} \frac{\Psi_{i}(x)-\Psi_{i}(y)}{|x-y|} \\
\quad G\left(\frac{\varphi_{1}(x)-\varphi_{1}(y)}{|x-y|}, \ldots, \frac{\varphi_{k}(x)-\varphi_{k}(y)}{|x-y|}\right) k(x, x-y) f(y) d y \mid
\end{aligned}
$$

is finite a.e. and belongs to $L^{\gamma}, 1 / q+\Sigma_{1}^{m} 1 / p_{i}=1 / \gamma$ provided that: (i) $k(x,-z)$ $=(-1)^{m+1} k(x, z)$, (ii) $G\left(W_{1}, \ldots, W_{k}\right)=G\left(-W_{1}, \ldots,-W_{k}\right)$, (iii) If $G\left(W_{1}, \ldots, W_{k}\right)$ is analytic in $\left|W_{j}\right|<R_{j}$ then $\left\|\nabla \varphi_{j}\right\|<c R_{j} / k$ where $c$ is a positive universal constant.

$$
0<\frac{1}{q}+\Sigma \frac{1}{p_{i}} \leqslant \frac{s-1}{s}+\frac{m}{n}, \quad q \geqslant \frac{s}{s-1}, p_{i}>1 .
$$

If $k(x, z)=k(z)$ we may take $s=1$ and condition (iv) is replaced by:

$$
0<\frac{1}{q}+\Sigma \frac{1}{p_{i}} \leqslant 1+\frac{m}{n}, \quad q>1, p_{i}>1 \text {. }
$$

REMARK. If we replace in (iv) $p_{i}>1$ by the weaker assumption $p_{i} \geqslant 1$ or in (v) $p_{i}>1, q_{i}>1$ by $p_{i} \geqslant 1, q_{i} \geqslant 1$, then $\stackrel{*}{T}$ belongs to weak $L^{\gamma}$ instead. 
Dirichlet and Neumann problems in $C^{1}$ domains. Let $\Omega \subset \mathrm{R}^{n}$ be a domain (open, bounded, and connected) with $C^{1}$ boundary, $\partial \Omega$. Assume $R^{n} \backslash \bar{\Omega}$ is also connected. In $\Omega$ we consider

(1) the Dirichlet problem, $\Delta u=0$ in $\Omega,\left.u\right|_{\partial \Omega}=g$ and

(2) the Neumann problem, $\Delta u=0$ in $\Omega,\left.\partial_{\eta} u\right|_{\partial \Omega}=g$, where $\partial_{\eta}$ denotes the normal derivative at the boundary. Using the results of [2] and the previous section we resolve (1) and (2) in the form of a double and single layer potential respectively. This classical approach reduces the question of existence to the study of the continuity and invertibility of the corresponding integral operators on the boundary.

For the Dirichlet problem we have the following result:

TheOREM 5. Suppose $g \in L^{p}(\partial \Omega), 1<p<\infty$, and let $N_{Q}$ denote the unit inner normal to $\partial \Omega$ at $Q$. Then there exists $\delta_{0}>0$, depending only on $\Omega$, and there exists a unique harmonic function, $u(X)$, defined in $\Omega$, such that for any $\alpha, 0<\alpha<1$,

(i) the function $u^{*}(Q)=\sup \left\{|u(X)|:|X-Q|\left\langle\delta_{0},\left\langle X-Q, N_{Q}\right\rangle \geqslant\right.\right.$ $\alpha|X-Q|\}$ belongs to $L^{p}(\partial D)$, and $\left\|u^{*}\right\|_{L p(\partial \Omega)} \leqslant C_{p}\|g\|_{L p(\partial \Omega)}$,

(ii) $u(X) \rightarrow g(Q)$ pointwise for almost every $Q$ as $X \stackrel{Q}{\rightarrow}$ and $\langle X-Q$, $\left.N_{Q}\right\rangle \geqslant \alpha|X-Q|$.

The solution of the Dirichlet problem in $C^{1}$ domains with $L^{p}$ data, $1<p$ $<\infty$, was first found by B. Dahlberg in [8] using the Poisson kernel for the domain. Indeed, in the same work Dahlberg solved the Dirichlet problem in any Lipschitz domain provided the data belongs to $L^{p}(\partial \Omega), 2 \leqslant p<\infty$.

The next result concerns regularity properties of the solution to the Dirichlet problem. First we define $L_{1}^{p}(\partial \Omega)$ as the space of functions in $L^{p}(\partial \Omega)$ whose first distribution derivatives (in local coordinates) are functions in $L^{p}(\partial \Omega)$. We define

$$
\|f\|_{L_{1}^{p}(\partial \Omega)}=\|f\|_{L^{p}(\partial \Omega)}+\|\nabla f\|_{L^{p}(\partial \Omega)} \cdot
$$

(See [9] for details about $L_{1}^{p}(\partial \Omega)$.)

THEOREM 6. Let $g \in L_{1}^{p}(\partial \Omega), 1<p<\infty$. For the solution $u$ of the Dirichlet problem with data $g$, and for $\delta_{0}$ and $\alpha$ as in Theorem 5 , set $(\nabla u)^{*}(Q)=$ $\sup \left\{\left|\nabla_{X} u(X)\right|:|X-Q|<\delta_{0},\left\langle X-Q, N_{Q}\right\rangle \geqslant \alpha|X-Q|\right\}$. Then

$$
(\nabla u)^{*} \in L^{p}(\partial \Omega) \text { and }\|(\nabla u) *\|_{L}^{p}(\partial \Omega) \leqslant C_{p}\|g\|_{L_{1}^{p}(\partial \Omega)} \cdot
$$

For the Neumann problem we obtain the following result.

TheOREM 7. Given $g \in L^{p}(\partial \Omega), 1<p<\infty$, there exists $u$, harmonic in $\Omega$, such that

$$
\|(\nabla u) *\|_{L} p_{(\partial \Omega)} \leqslant C_{p}\|g\|_{L^{p}(\partial \Omega)} \text { and }\left\langle\nabla u(X), N_{Q}\right\rangle \rightarrow g(Q)
$$


290 A. P. CALDERón, C. P. CALDERón, E. FABES, M. JODEIT AND N. M. RIVIERE

pointwise for almost every $Q$ as $X \rightarrow Q$ and $\left\langle X-Q, N_{Q}\right\rangle \geqslant \alpha|X-Q|$. $\left((\nabla u)^{*}\right.$ is defined as in Theorem 6.) Moreover the only solutions with zero Neumann data in the class of harmonic functions such that $\|(\nabla u) *\|_{L P(\partial \Omega)}<\infty$ are the constant functions.

\section{REFERENCES}

1. A. P. Calderón, Commutators of singular integral operators, Proc. Nat. Acad. Sci. U.S.A. 53 (1965), $1092-1099$.

2. Cauchy integrals on Lipschitz curves and related operators, Proc. Nat. Acad. Sci. U.S.A. 74 (1977),

3. A. P. Calderón and A. Zygmund, Local properties of solutions of elliptic partial differential equations, Studia Math. 20 (1961), 171-225.

4. C. P. Calderón, On commutators of singular integrals, Studia Math 53 (1975), 139

5. - On singular integral, Studia Math. (to appear).

6. R. R. Coifman, and Y. Meyer, On commutators of singular integrals and bilinear singular integrals, Trans. Amer. Math. Soc. 212 (1975), 315-332.

7. - Commutators d'integrals singulieres, Analyse Harmonique d'Orsay, No. 211, Université Paris XI, (1976).

8. Björn E. J. Dahlberg, On the Poisson integral for Lipschitz and $\boldsymbol{C}^{1}$ domains, Tech. report No. 1977-8, Dept. of Math., Chalmers Univ. of Tech. and Univ. of Göteborg (Preprint). 658-690.

9. R. T. Seeley, Singular integrals on compact manifolds, Amer. J. Math. 81 (1959),

DEPARTMENT OF MATHEMATICS, UNIVERSITY OF CHICAGO, CHICAGO, ILLINOIS, 60637

DEPARTMENT OF MATHEMATICS, UNIVERSITY OF ILLINOIS AT CHICAGO CIRCLE, CHICAGO, ILLINOIS 60680

DEPARTMENT OF MATHEMATICS, UNIVERSITY OF MINNESOTA, MINNEAPOLIS, MINNESOTA 55455 